

DOI: http://doi.org/10.48195/jie2021-110

\title{
OS EFEITOS DA GOLDEN HOUR NA SAÚDE DO RECÉM-NASCIDO A TERMO ${ }^{1}$
}

\section{Dayane do Amaral Rodrigues ${ }^{2}$; Fabiani Gonzaga Noronha ${ }^{3}$; Lenise Dutra da Silva ${ }^{4}$; Thais Jaíne Rorato ${ }^{5}$; Flávia Camef Dorneles Lenz ${ }^{6}$; Bibiana Antunes ${ }^{7}$}

\begin{abstract}
RESUMO
Este estudo teve por objetivo descrever sobre as produções científicas relacionadas aos efeitos da Golden hour na saúde do recém-nascido a termo. Trata-se de uma revisão integrativa, cujas buscas foram realizadas nas bases de dados Medical Literature Analysis and Retrieval System Online e PubMed, no mês de julho de 2020, com a análise de três artigos. A Golden hour é um método utilizado nos primeiros sessenta minutos de vida do neonato, visando minimizar acometimentos neonatais. $\mathrm{O}$ contato pele a pele, a amamentação e o vínculo inicial proporcionam o desenvolvimento saudável do bebê a longo prazo. Porém emergiram poucos estudos nesta busca que discutam essa temática com as instituições e profissionais de saúde. Apresentando assim uma grande dificuldade de entendimento profissional e também das mulheres parturientes devido a desinformação dos benefícios com a aplicação da hora ouro, além da resistência dos profissionais de saúde.
\end{abstract}

Palavras-chave: Neonato; Hora dourada; Pesquisa.

\begin{abstract}
This study aimed to describe the scientific productions related to the effects of Golden hour on the health of newborns at term. It is an integrative review, whose searches were carried out in the databases Medical Literature Analysis and Retrieval System Online and PubMed, in July 2020, with the analysis of three articles. Golden hour is a method used in the first sixty minutes of a newborn's life, aiming to minimize neonatal disorders. Skin-to-skin contact, breastfeeding and the initial bond provide the baby with healthy long-term development. However, few studies have emerged in this

\footnotetext{
${ }^{1}$ Trabalho realizado na disciplina Saúde Neonatal do Curso de Pós-Graduação Saúde Materna e Neonatal da Universidade Franciscana.

${ }^{2}$ Psicóloga. Especialista em Saúde Materna e Neonatal da Universidade Franciscana. dayaneamaralr@gmail.com

${ }^{3}$ Terapeuta Ocupacional. Especialista em Saúde Materna e Neonatal da Universidade Franciscana. fg.terapeutaocupacional@gmail.com

${ }^{4}$ Enfermeira. Mestre em Saúde Materno Infantil. Especialista em Saúde Materna e Neonatal da

Universidade Franciscana. Docente do Curso de Enfermagem da URI-Santiago. enf.lenise@gmail.com

${ }^{5}$ Enfermeira. Especialista em Gestão Hospitalar. Especialista em Saúde Materna e Neonatal da Universidade Franciscana. thais1994rorato@yahoo.com.br

${ }^{6}$ Enfermeira. Graduada pela Universidade Regional Integrada do Alto Uruguai e das Missões- Campus Santiago. flaviacamefd@gmail.com

${ }^{7}$ Enfermeira. Doutoranda do Programa de Pós-graduação da UFRGS. bibianaantunes@ @otmail.com
} 


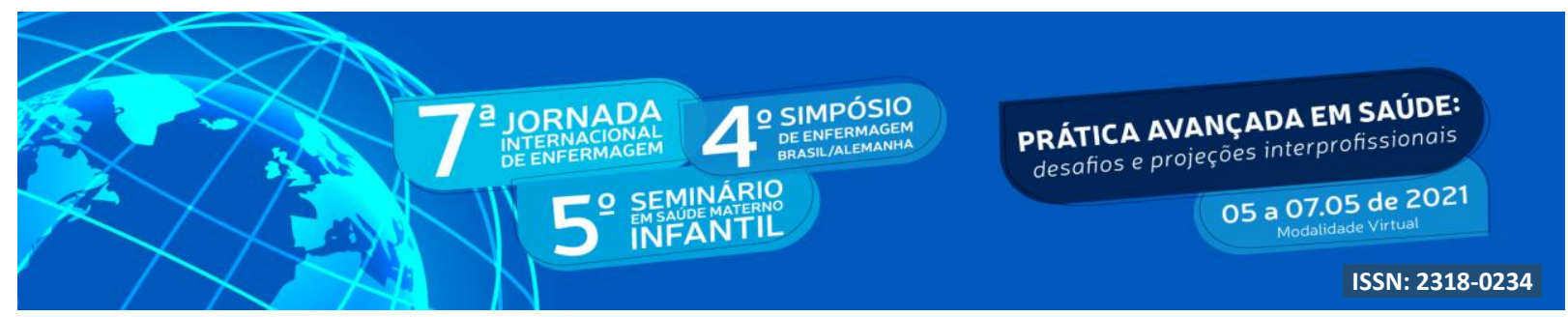

search that discuss this issue with health institutions and professionals. Thus presenting a great difficulty of professional understanding and also of the parturient women due to the misinformation of the benefits with the application of the golden hour, besides the resistance of the health professionals.

Keywords: Neonate; Golden hour; Search.

\section{INTRODUÇÃO}

A humanização do parto e nascimento a muitos anos vem sendo discutida e apresentada de várias formas. No século XX, a humanização era compreendida como a minimização da dor do parto, a partir de medicamentos sedativos, alucinógenos e indutores e na realização de episiotomia, uso de fórceps e outras tecnologias invasivas. O parto era vistocomo um momento fisiologicamente patogênico e que necessitava de intervenções (BRENES, 1991; MENDONÇA, 2015, DINIZ, 2005).

Com o passar dos tempos o parto se tornou um procedimento a ser realizado nos hospitais e não mais nos domicílios e casas de parto. Neste período a mulher podia optar entre ter um parto sem sofrimento a partir da cesárea eletiva ou um parto normal induzido com o uso de ocitócitos, episiotomia e fórceps, realidade encontrada até os dias atuais em muitos hospitais brasileiros (DINIZ, 2005; LEAL et al., 2014).

A utilização das tecnologias de forma inapropriada e rotineira levou ao aumento da morbimortalidade materna e perinatal em todo o mundo, principalmente em países subdesenvolvidos. Em 2000, a Organização das Nações Unidas (ONU) listou os maiores problemas mundiais e propôs metas para tornar o mundo melhor até 2015, conhecido como Objetivos de Desenvolvimento do Milênio (ODM). Nessas metas estavam incluídas a redução da mortalidade infantil e a melhoria à saúde das gestantes (DIAS, 2011; ONU, 2015).

Nesse contexto, o Brasil desenvolveu iniciativas para atender as metas, como a Rede Cegonha, Política Nacional de Atenção Integral à Saúde da Mulher e o Pacto Nacional pela Redução da Mortalidade Materna e Neonatal. Com a implantação dessas estratégias houve uma redução dos óbitos de gestantes de 55\%, 


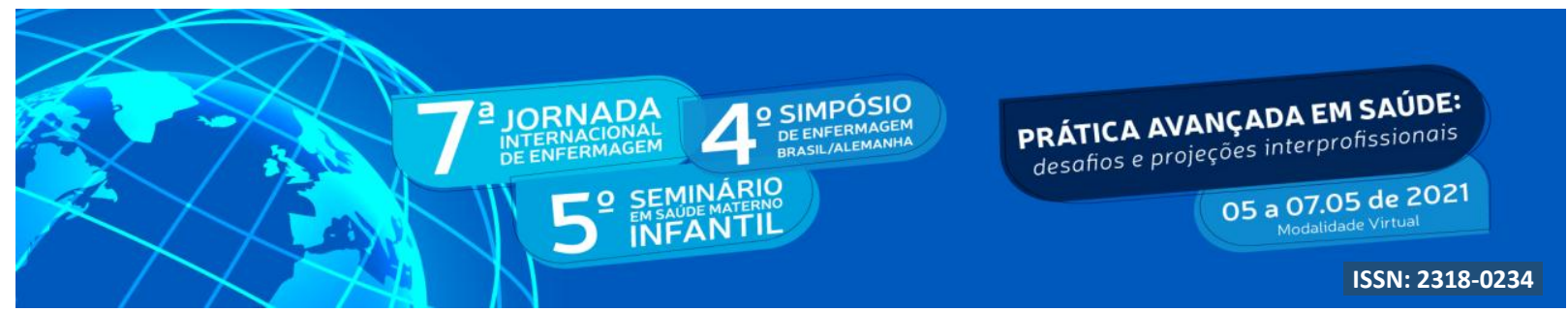

porém não se atingiu a meta estipulada para a redução da mortalidade materna (ONU, 2015; FIOCRUZ, 2010).

Compreende-se que para a diminuição da mortalidade materna e perinatal sejam realizadas algumas condutas durante o trabalho de parto e pós-parto, dentre elas o contato pele a pele, estimulo a amamentação na primeira hora de vida do $\mathrm{RN}$ e o clampeamento oportuno do cordão umbilical, período denominado Golden Hour ou hora dourada (ARRUDA et al., 2018; BRASIL, 2014).

O contato pele a pele é uma prática assegurada pelo Ministério da Saúde (MS) a todos os recém-nascidos de baixo risco, pois se apresenta como um procedimento seguro, de baixo custo e com comprovados benefícios a curto e longo prazo à saúde materna e neonatal. Quando se realiza o contato da mãe com o bebê comportamentos neurofisiológicos são despertados no organismo materno, o qual estimula a produção dos hormônios prolactina e ocitocina. Este processo permite que o RN receba na primeira hora de vida o colostro e que o corpo da mãe inicie a produção de leite e sua posterior ejeção. Ainda, a amamentação na primeira hora pós-parto auxilia na contração uterina, diminuindo o risco de hemorragia puerperal e fortalecendo o vínculo mãe-bebê (OPAS, 2001; ARAÚJO, 2018; BRASIL, 2014).

Outra prática da Golden hour é o clampeamento tardio do cordão umbilical, quando realizado em RNs de baixo risco e sem contraindicação, favorece a adaptação fisiológica do recém-nascido a vida extrauterina e diminui o risco de anemia ferropriva até os seis primeiros meses de vida (GOES, 2017).

\section{OBJETIVO}

Descrever sobre as produções científicas relacionadas aos efeitos da Golden hour na saúde do recém-nascido a termo.

\section{METODOLOGIA}




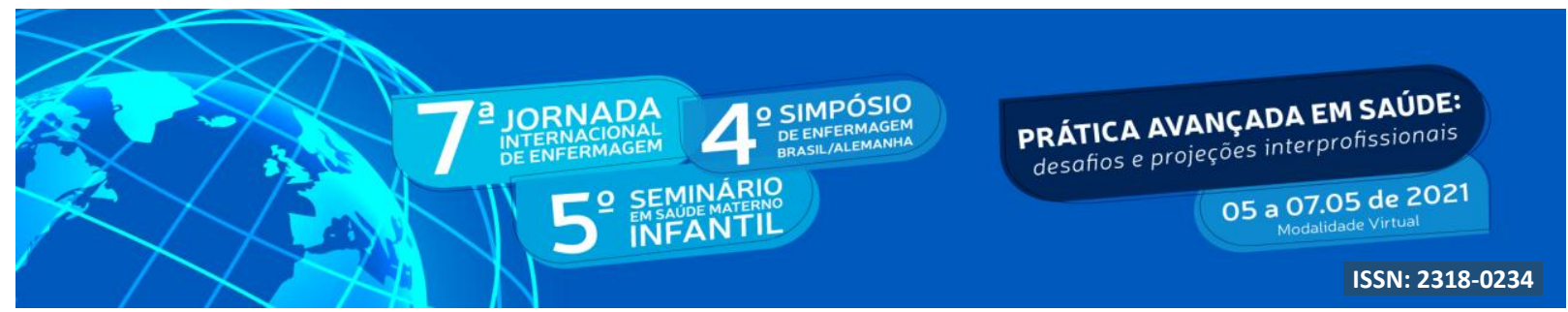

Trata-se de uma revisão integrativa da literatura científica, cujos periódicos foram consultados no mês de julho de 2020. A revisão integrativa é uma metodologia que propicia a análise, interpretação e síntese de estudos experimentais ou não-experimentais pré-existentes. Este método possibilita que sejam realizadas conclusões gerais a respeito de uma temática e promove discussões críticas a respeito do objetivo, método e resultados das pesquisas já realizadas (WHITTEMORE; KNALF, 2005; BROOME, 1993). Para a elaboração desta revisão integrativa foram seguidas as seis fases apresentadas na sequência (GANONG, 1987).

A primeira fase constituiu da formulação da pergunta norteadora "Quais são os efeitos da Golden hour na saúde do recém-nascido a termo?" Para responder esta pergunta realizaram-se buscas nas bases de dados Medical Literature Analysis and Retrieval System Online (Medline) acessada a partir do formulário "Busca Avançada" do Portal Regional da Biblioteca Virtual em Saúde (BVS) e na PubMed. Na segunda fase foram definidos como critérios de inclusão: artigos disponíveis na íntegra, em idiomas português, inglês e espanhol, publicados e indexados nas referidas bases de dados e publicados nos últimos cinco anos. Como critérios de exclusão: artigos completos não gratuitos, revisões, dissertações, teses e editoriais. Estudos encontrados em mais de uma base de dados foram considerados somente uma vez.

A terceira e quarta fases se constituíram da busca na base a partir das palavras-chave "Golden hour" and "neonato" e mesh "Golden hour" and "neonate". Nestas fases foram encontrados 254 artigos, dos quais se realizou a leitura dos títulos e resumos e, na sequência, a leitura na íntegra dos artigos que apresentavam conteúdo concernente ao objetivo proposto.

A amostra final deste processo foi constituída de cinco artigos. A posteriori, se criou um fluxograma com o detalhamento das fases de pesquisa (Figura 1) e uma tabela contendo a síntese nos artigos selecionados (Tabela 1) e o nível de evidência científica. Já, na quinta fase, realizou-se a interpretação dos resultados; e, na sexta fase, a síntese do conhecimento, com a análise dos vieses. 


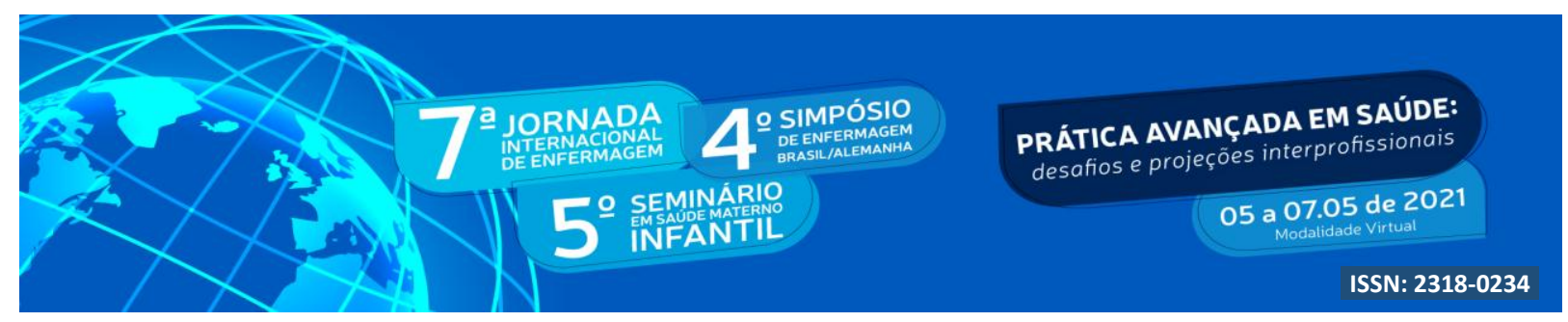

Figura 1- Fluxograma com o detalhamento da pesquisa

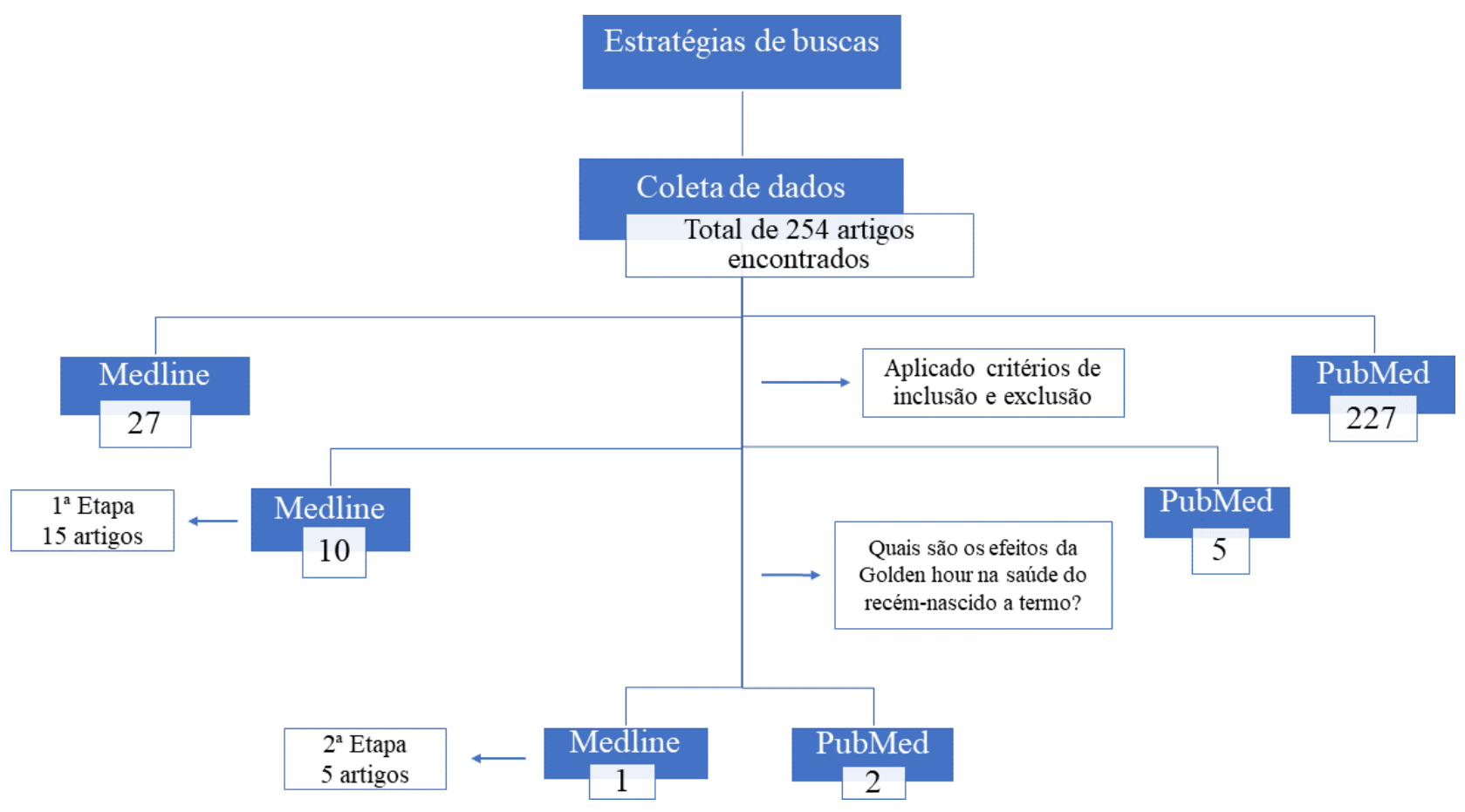

Fonte: Elaborada pelas autoras.

\section{RESULTADOS E DISCUSSÃO}

Após a análise criteriosa dos artigos selecionados na íntegra, os mesmos foram apresentados em forma de tabela, conforme se segue (Tabela 1). 


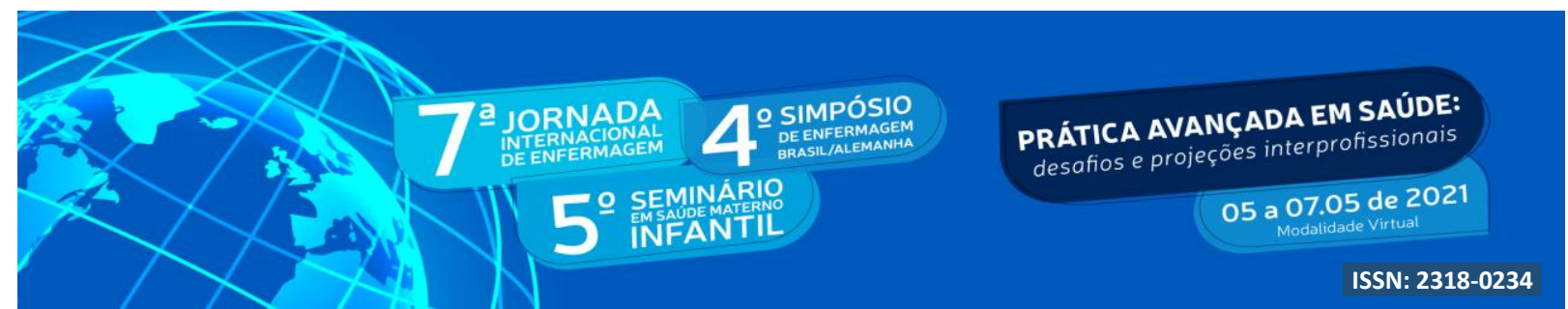

Tabela 1- Caracterização dos artigos selecionados e analisados na revisão integrativa, Brasil, $2020(\mathrm{~N}=3)$

\begin{tabular}{|c|c|c|c|c|c|c|}
\hline & $\begin{array}{c}\text { Base de } \\
\text { dados }\end{array}$ & Autores & Título & Ano/ Periódico & Objetivo & $\begin{array}{c}\text { Tipo de } \\
\text { pesquisa }\end{array}$ \\
\hline Al & PUBMED & CRENSHA, W. J & $\begin{array}{l}\text { Healthy Birth Practice } \\
\text { \#6: Keep Mother and } \\
\text { Newborn Together- } \\
\text { It's Best for Mother, } \\
\text { Newborn, and } \\
\text { Breastfeeding. }\end{array}$ & $\begin{array}{c}2019 / \text { The jornal } \\
\text { of perinatal } \\
\text { education }\end{array}$ & - & $\begin{array}{l}\text { Updated } \\
\text { evidence- based } \\
\text { review }\end{array}$ \\
\hline A2 & MEDLINE & $\begin{array}{l}\text { NECZYPO, R. J. } \\
\text { L; HOLLEY, S. L }\end{array}$ & $\begin{array}{l}\text { Providing Evidence- } \\
\text { Based Care During the } \\
\text { Golden Hour }\end{array}$ & $\begin{array}{l}\text { 2017/Nursing } \\
\text { for Women's } \\
\text { Health }\end{array}$ & $\begin{array}{l}\text { Explores the evidence } \\
\text { supporting the Golden hour and } \\
\text { provides strategies for } \\
\text { successfully implementing a } \\
\text { Golden hour protocol ona } \\
\text { hospital-based labor and } \\
\text { delivery unit }\end{array}$ & Exploratory \\
\hline $\mathbf{A 3}$ & PUBMED & $\begin{array}{c}\text { NIELA-VILEN, } \\
\text { H.; AXELINA, } \\
\text { A.; FLACKIN } \\
\text { G, R. }\end{array}$ & $\begin{array}{l}\text { The golden hour } \\
\text { in Finnish birthing } \\
\text { units - An } \\
\text { ethnographic study }\end{array}$ & $\begin{array}{l}\text { 2020/ } \\
\text { Elsevier }\end{array}$ & $\begin{array}{l}\text { The objective of this study was } \\
\text { to explore midwives and } \\
\text { parents percepti ons and actions } \\
\text { as well as the culture } \\
\text { surrounding the golden hour in a } \\
\text { birthing room. }\end{array}$ & $\begin{array}{c}\text { Ethnographic } \\
\text { approach }\end{array}$ \\
\hline
\end{tabular}

A Golden hour é um método utilizado nos primeiros sessenta minutos de vida do neonato, visando minimizar acometimentos neonatais. Nesta abordagem clínica, o contato pele a pele, a amamentação e o vínculo inicial proporcionam o desenvolvimento saudável do bebê (Reynolds et al., 2009; Silva et al, 2016).

Diante disso, A1 demonstram em seus estudos os benefícios da Golden hour na saúde materna e neonatal a curto e longo prazo. Foi possível perceber que promover esse momento é uma responsabilidade da equipe e que deve ser apoiado e incentivado em todas as maternidades. A autora ainda destaca que as mulheres devem ser orientadas quanto aos benefícios do contato pele a pele e da amamentação na primeira hora pós-parto e os riscos da não realização (CRENSHAW, 2019).

Compreende-se que toda puérpera e RN sem complicações, têm o direito de não serem separados após o nascimento, preferencialmente na primeira hora após o parto. Entende-se que durante esse período o $\mathrm{RN}$ deve ser colocado em contato pele a pele com sua mãe, sem a presença de barreiras (cobertores ou roupas) e coberto com campos estéreis, limpos, secos e aquecidos. Ainda, durante esse período podem ser realizados os cuidados de rotina, postergando os procedimentos que não são urgentes como os dados antropométricos (WHO; 


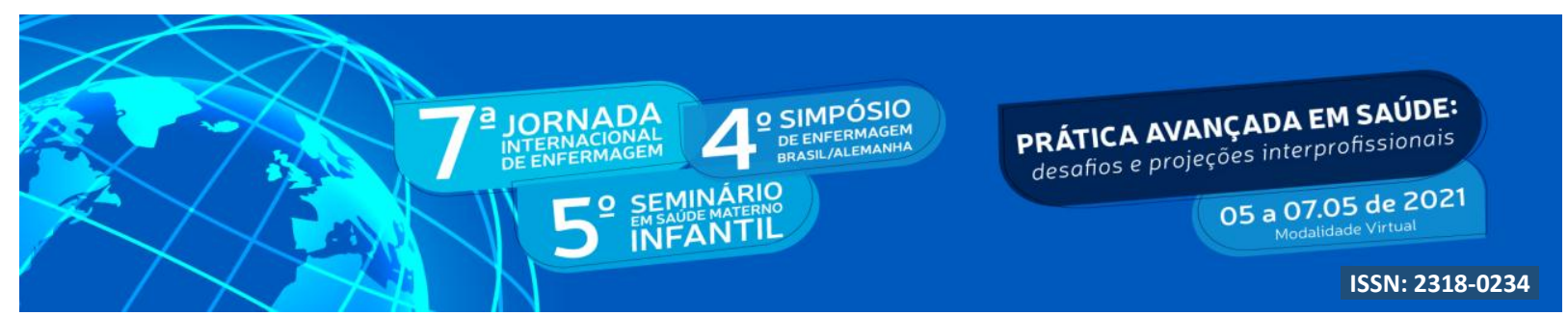

UNICEF, 2018).

Diferentemente dos compilados de A1 e A3, o estudo de A2 abordou que a prática da Golden Hour pode estimular o clampeamento tardio do cordão umbilical, aumentando os índices de amamentação assim como facilitando o vínculo entre mãe e bebê pelo contato pele a pele (NECZYPOR; HOLLEY, 2017). Acrescentam Cortés et al. (2015) que entre os vários benefícios da prática do clampeamento tardio do cordão umbilical, está o aumento das reservas de ferro no bebê. Ainda, a realização do plano de parto (PP) pelas gestantes no prénatal, contribui para as práticas de parto humanizado.

No estudo de A3, não foi possível observar se era realizado o contato pele a pele e o estímulo a amamentação, no entanto, ocorria nos primeiros cinco a sete minutos após o nascimento imediato como preconizado. Percebe-se a inflexibilidade da equipe na realização da Golden Hour. A realização do clampeamento oportuno do cordão ocorria com o RN sobre a cama e após era colocado em contado com a mãe (NIELA-VILEN; AXELINA.; FLACKING, 2020). Neste estudo, percebe-se o quanto ainda se encontram dificuldades na implementação da Golden Hour nos primeiros minutos de vida. Para Matos et al. (2010) os profissionais de saúde executam um papel determinante na realização deste contato, porém, a realização desta prática de forma mecânica simplesmente para cumprir um protocolo institucional transforma este momento em um breve contato, em que a mulher não possui autonomia suficiente para exercer o papel de protagonista (SANTOS et al., 2013).

Outra questão observada na literatura de A1, foi que o contato pele a pele deve ser incentivado tanto no parto normal quando no parto cesáreo, visto os muitos benefícios que têm. Estudos demonstraram que quando realizado durante a cesariana a mulher apresenta redução da dor, pois se concentra no RN, minimizando a internação de RNs em Unidade de Terapia Intensiva Neonatais (SCHNEIDER; CRENSHAW; GILDER, 2017; SUNDIN; MAZAC, 2015).

Com os desfechos dos artigos A1, A2 e A3, ressalta-se que a não realização da Golden hour parte do desconhecimento da equipe sobre os efeitos positivos à saúde materna e neonatal. Salienta-se que quando não ofertado 


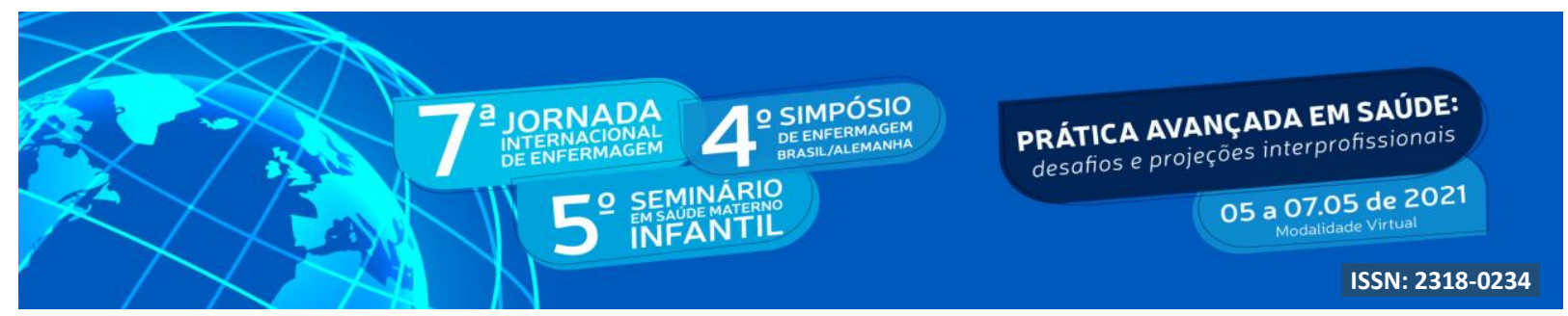

esse momento ao binômio estes têm efeitos nocivos à saúde psicofisiológica. O contato pele a pele deve ser sempre que possível incentivado, pois apresenta impactos positivos além do momento do nascimento. Dentre esses impactos estão a diminuição do risco para depressão pós-parto, desenvolvimento de sentimentos materno como segurança, confiança e capacidade para realizar os cuidados com o $\mathrm{RN}$, bem como o $\mathrm{RN}$ apresenta redução nos níveis de estresse, conseguindo dormir melhor, aspecto importante para o seu crescimento e desenvolvimento saudável (MORGAN; HORN; BERGMAN, 2011).

\section{CONCLUSÃO}

Constatou-se neste estudo a importância da aplicação das práticas da Gold hour na primeira hora de vida do recém-nascido e da mãe. Trazendo inúmeros benefícios para ambos como diminuição da morbidade materna e neonatal, promover vínculo entre mãe/bebe e também o aumento das taxas de aleitamento materno, entre outros ganhos. Essas necessidades fisiológicas e emocionais que mães e bebes apresentam após o nascimento, originarão a promoção em saúde a longo prazo através de pequenas atitudes vindas dos profissionais que ali então a auxiliando.

Desta forma, apesar da reconhecida importância de todas vantagens que a Gold hour trás para a mulher e o recém-nascido, emergiram poucos estudos para discutir essa temática com as instituições e profissionais de saúde. Apresentando assim uma grande dificuldade de entendimento profissional e também das mulheres parturientes devido a desinformação dos benefícios com a aplicação da hora ouro, acarretando a não implementação entre as rotinas hospitalares nas unidades de saúde. Além disso, ainda, observou-se a resistência profissional em apoiar essas atividades.

Considera-se como limitações deste estudo a escolha por apenas três idiomas e artigos gratuitos dos últimos cinco anos. Sugere-se que sejam realziadas novas pesquisas com mais inclusões e que sejam divulgadas pelas instituições e pelos profissionais de saúde suas experiências na aplicação da Golden Hour e os impactos na saúde materna e neonatal. 


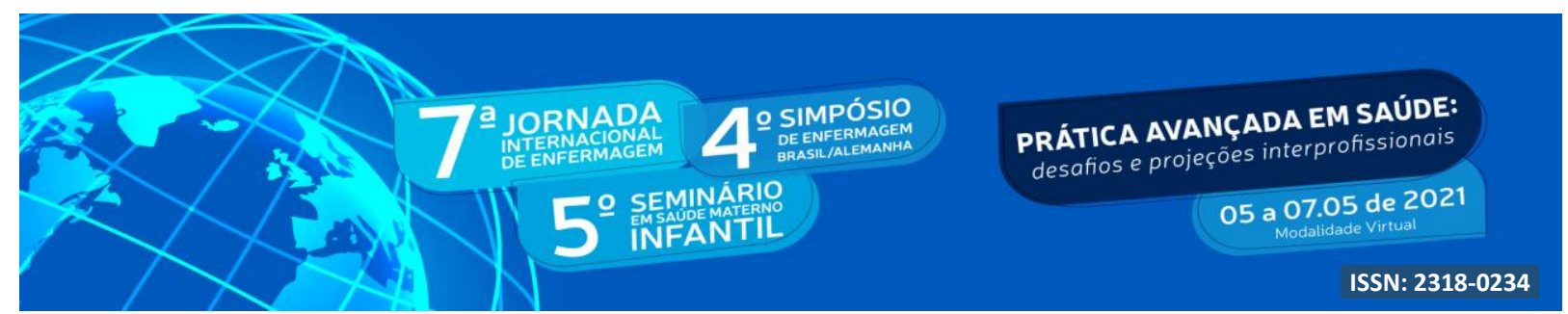

\section{REFERÊNCIAS}

ARAÚJO, A. L. Cuidado de Enfermagem ao recém-nascido prematuro na UTI Neonatal. Trabalho de Conclusão de Curso (Enfermagem) -Universidade Anhanguera, Osasco/SO, 2018.

ARRUDA, G.T. et al. Existe relação da via de parto com a amamentação na primeira hora de vida? Revista Brasileira em Promoção da Saúde. v. 31, n. 2, p. 1-7, 2018.

BOCCOLINI CS, CARVALHO ML, OLIVEIRA MIC, VASCONCELLOS AGG. Fatores associados à amamentação na primeira hora de vida. Rev Saúde Pública. v.45 n.1 São Paulo, 2010 .

BRASIL. Ministério da Saúde. Secretaria de Atenção à Saúde. Portaria nº 371, de 7 de maio de 2014. Institui diretrizes para a organização da atenção integral e humanizada ao recém nascido (RN) no Sistema Único de Saúde(SUS). Diário Oficial da União, Brasília, DF, 8 de maio de 2014.

BRENES, A. C. História da parturição no Brasil, século XIX. Cad. Saúde Pública v.7, n.2, Rio de Janeiro,1991.

BROOME, M. E. Revisão integrativa da literatura para o desenvolvimento de conceitos. Em: Rodgers BL, Knafl KA. Desenvolvimento de conceito em enfermagem. Filadélfia: Saunders; p. 231-50, 1993.

DIAS, M. A. B. Humanização do parto: política pública, comportamento organizacional e ethos profissional. Cad. Saúde Pública, Rio de Janeiro, v. 27, n. 5, p. 1042-1043, Mai, 2011. Disponível em <http://www.scielo.br/scielo.php?script=sci_arttext\&pid=S0102311X2011000500022\&lng=en\&nrm=iso>. Acesso em 07 de julho de 2020.

DINIZ, C. S. G. Humanização da assistência ao parto no Brasil: os muitos sentidos de um movimento. Ciência \& Saúde Coletiva. v. 10, n. 3, p. 627-637, 2005.

FLACKING, R. et al. Closeness and separation in neonatal intensive care. Acta Paediatrica, Malden, v. 101, n. 10, p. 1032-1037, 2012.

Fundação Oswaldo Cruz - Fiocruz. Objetivos de Desenvolvimento do Milênio - ODM.

Disponível em: < http://www.fiocruz.br/omsambiental/media/ODMBrasil.pdf> Acesso em 07 de julho de 2020.

GANONG, L. H. Revisões integrativas da pesquisa em enfermagem. Res Enfermeira Saúde. v. 10, n. 1, p. 1-11, 1987.

GOES, J. F. Clampeamento tardio do cordão umbilical: Estudo de corte. Dissertação de Mestrado apresentado ao Programa de Pós-graduação da Saúde da Criança de da Mulher. Rio de Janeiro, 2017. 


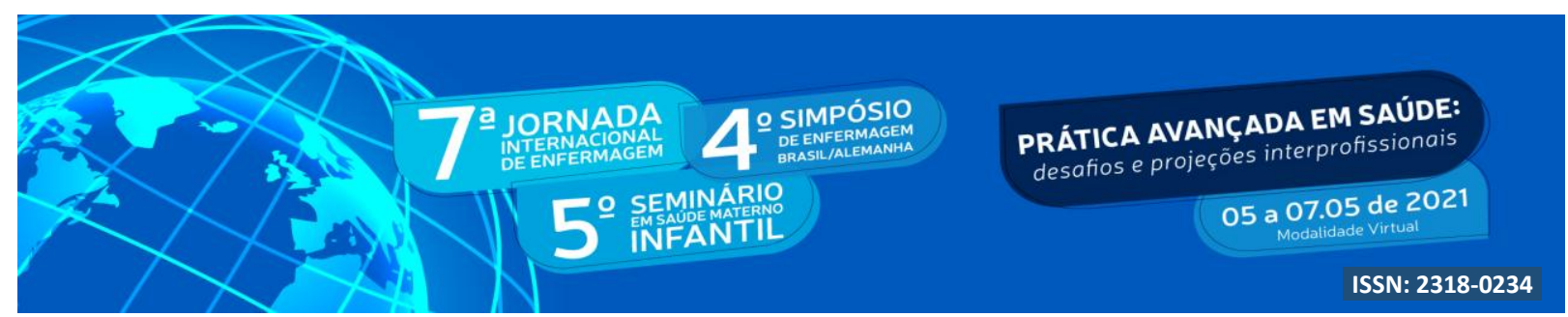

LEAL, M. do C. et al. Intervenções obstétricas durante o trabalho de parto e parto em mulheres brasileiras de risco habitual. Cadernos de Saúde Pública, [s.l.], v. 30, n. 1, p.17-32, ago, 2014.

MARQUES, A.N. O que diz a literatura sobre contato pele a pele entre a mãe e o recémnascido durante a cesariana: em busca de argumentos para as boas práticas na atenção ao nascimento. Trabalho de Conclusão de Curso (Enfermaem). UFMG/ UFRGS, 2016.

MENDONÇA, S. S. Modelos de assistência obstétrica concorrentes e ativismo pela humanização do parto. Civitas, Rev. Ciênc. Soc. v.15, n.2, Porto Alegre, 2015.

MATOS, T.A et. al. Contato precoce pele a pele entre mãe e filho: significado para mães e contribuições para a enfermagem. Revista Brasileira de Enfermagem, Brasília, v.63, n.6, p.998-1004, nov/dez, 2010.

MENDONÇA, S. S. Modelos de assistência obstétrica concorrentes e ativismo pela humanização do parto. Civitas, Rev. Ciênc. Soc. v.15, n.2, Porto Alegre, 2015.

ORGANIZAÇÃO DAS NAÇÕES UNIDAS. PNUD explica transição dos Objetivos do Milênio aos Objetivos de Desenvolvimento Sustentável. Nações Unidas Brasil, 2015. 11

Disponível em: < https://nacoesunidas.org/pnud-explica-transicao-dos-objetivos-domilenioaos-objetivos-de-desenvolvimento-sustentavel/> Acesso em: 07 de julho de 2020.

ORGANIZAÇÃO PANAMERICANA DE SAÚDE. Evidências científicas dos dez passos para o sucesso do aleitamento materno. Brasília: OPAS, 2001.

REYNOLDS RD, PILCHER J, RING A, JOHNSON R, MCKINLEY P. The Golden Hour: care of the LBW infant during the first hour of life one unit's experience. Neonatal Netw. 28(4):211-19, 2009.

SANTOS G.N. et al. Assistência ao recémnascido na sala de parto sob a ótica das puérperas. Rev Interdiscip. 6(1):43-51, 2013.

FONSECA MCC. Vivenciando o contato pele a pele com o recém-nascido no pós-parto como um ato mecânico. Rev Bras Enferm. 67(2):202-07, 2014.

SILVA CM, PEREIRA SCL, PASSOS IR, SANTOS LC. Fatores associados ao contato pele a pele entre mãe/filho e amamentação na sala de parto. Rev Nutr. 29(4):457-71 2016.

WHITTEMORE, R.; KNAFL, K. The integrative review: update methodology. J Adv Nurs. v. 52 , n. 5, p. 546-53, 2005 